\title{
Post-AGB Evolution
}

\author{
Hans Van Winckel \\ Instituut voor Sterrenkunde, K.U. Leuven, Celestijnenlaan 200B, \\ 3001 Heverlee, Belgium
}

\begin{abstract}
.
This review focuses on the evolution of single and binary optically bright post-AGB stars and the relation between the photospheric chemical abundances and the circumstellar material.

Surface abundance determinations of optically bright post-AGB stars offer a powerful tool, not only to clarify the evolutionary nature of the objects themselves, but also to constrain theoretical calculations for chemical evolution. However, a survey of recent results shows that the chemical patterns observed are very diverse and that several different classes can be distinguished. The most striking result is that only a minor fraction, namely the $21 \mu \mathrm{m}$ objects, are conform to standard post third dredge-up theory.

In recent years it became clear that binarity can influence the late evolutionary stages considerably. A second part therefore discusses the varied and fundamental impact of the presence of a companion on the evolution of post-AGB systems.
\end{abstract}

\section{Introduction}

Post-AGB stars, also commonly named proto-planetary nebulae, are generally defined as objects having evolved off the AGB but not (yet) to high enough effective temperatures to ionise a Planetary Nebula (PN). As direct descendants of AGB stars, they are not only interesting objects by themselves, but in principle also ideal tracers to gain better insight into the complex physical and chemical changes that occurred during the previous (late) AGB phase. This is, however, not straightforward since theoretical (chemical) evolutionary AGB predictions are not only dependent on fundamental parameters like initial mass and metallicity, but also on less well constrained parameters like mass-loss history and geometry, details of the dredge-up phenomenon and thermal pulse nucleosynthesis, interpulse phase at which the post-AGB phase starts etc. (see e.g. the contributions of Lattanzio \& Forestini, Blöcker, Mowlavi, Herwig et al. and Marigo in this volume). Moreover, since the post-AGB evolution is short (typically $10^{3}$ to $10^{4}$ years: e.g. Blöcker 1995), not many post-AGB stars are known and observational results on individual sources are rather difficult to interpret in a more general picture.

This review focuses on many recent observational results on optically bright post-AGB stars and tries to emphasise the most important findings in the general context of stellar evolution theory, together with some warning against observa- 
tional bias introduced by selection procedures. Optically bright objects have the extra advantage that the broad spectral domain of their emission allows us not only to study the stellar F-K type photospheres in detail, but also the complex physical and chemical interplay between the central star and the circumstellar material. Recent results on optically bright post-AGB stars experiencing a late thermal pulse will not be covered in this paper but I refer to the contributions of Asplund, Cohen et al., Yudin \& Tatarnikov and Werner et al. in this volume.

\section{SED: detached dust-shells versus broad IR excesses in binaries}

The IRAS Point Source Catalogue has been used extensively to search for new post-AGB stars (e.g. Pottash \& Parthasarathy 1988; Hrivnak et al. 1989; Oudmaijer et al. 1992). The Spectral Energy Distributions (SEDs) obtained for the optically bright post-AGB candidate stars fall into two categories: the first one contains objects showing a double-peaked energy distribution determined by the UV-optical emission of the reddened stellar photosphere and the thermal re-radiation of the circumstellar, detached, and expanding dust-shell with a characteristic dust temperature of $\mathrm{T}_{d u s t} \sim 100-200 \mathrm{~K}$ (for examples of SEDs e.g.: Hrivnak et al. 1989; Trams et al. 1991; Szczerba et al. 1997).

A significant fraction of post-AGB candidate stars also show the presence of a hot dust component with $\mathrm{T}_{d u s t} \sim 500-1300 \mathrm{~K}$ making the IR excess very broad (for examples of SEDs e.g.: Trams et al. 1991; Waters et al. 1993). Subsequent radial velocity measurements revealed a tight correlation between the occurrence of hot dust and binarity (Waters et al. 1997 and references therein). Since the binary nature of the object can have fundamental influence not only on the SED but also on the evolution of the whole object, we will cover the binary post-AGB stars in a separate section (Sect. 4). The next section will cover the double peaked post-AGB objects for which no binary motion has yet been discovered.

\section{3. (Single) Post-AGB evolution}

\section{1. $21 \mu \mathrm{m}$ objects as post-carbon stars}

The IR emission feature at $21 \mu \mathrm{m}$ was first identified on IRAS LRS spectra by Kwok et al. (1989) and it was realised that the feature only occurs in spectra of C-rich post-AGB stars as evidenced by the presence of PAH bands in the mid-IR and often strong HCN fluxes (e.g. Kwok et al. in this volume). The 21 $\mu \mathrm{m}$ feature is sometimes accompanied by a strong $30 \mu \mathrm{m}$ emission feature which is observed over a broader spectral type domain (Omont et al. 1995).

The carbon rich nature of the circumstellar environment of these objects was corroborated by the detection of $\mathrm{C}_{2}, \mathrm{C}_{3}$ and $\mathrm{CN}$ bands in the optical spectra (Hrivnak 1995). Detailed analysis of high-resolution spectra of these bands indicates that the ro-vibrational lines are formed in a relatively narrow shell in the circumstellar envelope (Bakker et al. 1996; 1997). Bakker \& Lambert (1998) estimated on the basis of ultra high-resolution spectroscopy of these bands a ${ }^{12} \mathrm{C} /{ }^{13} \mathrm{C}$ ratio of 67 for $\mathrm{HD} 56126$, demonstrating again an efficient 3rd dredge-up. 
Chemical studies of the stellar photospheres confirmed the post-3rd dredgeup character of known $21 \mu \mathrm{m}$ stars since they show not only a high $\mathrm{C} / \mathrm{O}$ ratio but also large overabundances of s-process elements (Klochkova 1995; Začs et al. 1995; Van Winckel et al. 1996; Reddy et al. 1997; Decin et al. 1998). But also the reverse still holds: all known post-AGB stars showing strong s-process enhancements in their optical spectra display a $21 \mu \mathrm{m}$ feature in their IR spectra (Decin et al. 1998). The F-G spectral types of these supergiants make them ideal objects to study a wide variety of species by their atomic photospheric lines, making them ideal sources to study the AGB nucleosynthesis and dredgeup phenomena. The sample of $21 \mu \mathrm{m}$ objects studied in detail till now displays a surprisingly large variety of integrated neutron exposures, as parameterised by the heavy-to-light s-process index ([hs/ls] value, see also Smith in this volume) in between -0.6 and +0.5 , independent on metallicity.

Most $21 \mu \mathrm{m}$ objects are clearly resolved at mid-IR wavelengths and show axial symmetry with a $\dot{\mathrm{M}}_{\text {equator }} / \dot{\mathrm{M}}_{\text {pole }}$ up to 20 (e.g. Meixner et al. 1997; Dayal et al. 1998). The resolved axial symmetry is certainly not limited to 21 $\mu \mathrm{m}$ objects and is in fact very common among double-peaked post-AGB stars. How this axial symmetry develops during the superwind phase is still a matter of debate (e.g. review by Meixner 1997) but is, however, fundamental since the future $\mathrm{PN}$ is shaped during this phase.

\subsection{Dredge-up efficiency versus metallicity}

The $21 \mu \mathrm{m}$ stars are, however, only a fraction of the double-peaked post-AGB stars and the chemical composition of other sources are much more difficult to interpret (Luck et al. 1990; Van Winckel 1997; Začs et al. 1996). To illustrate the dredge-up efficiency Fig. 1 shows the overabundance of the s-process element Zirconium $(\mathrm{Zr})$ as a function of the metallicity for a sample of homogeneously analysed objects. In other respects like IR emission, kinematics and metallicities these stars are similar, but there is a clear difference in photospheric composition. The absolute luminosity differences are of course very difficult to trace but may be crucial for understanding the different chemical evolutions.

This chemical diversity is emphasized further by the characteristic chemical patterns displayed by the hot B-type post-AGB stars. These optically bright post-AGB stars are not discovered by their IR properties but are identified among samples of high-galactic latitude B stars selected to study early-type stars in the halo of our Galaxy (e.g. Conlon et al. 1991; McCausland et al. 1992; Napiwotzki et al. 1994; Moehler \& Heber 1998). Although the Fe abundance is very difficult to trace for the hottest atmospheres, the estimated metallicities determined from other species $(\mathrm{Mg}, \mathrm{Al}, \mathrm{Si}$ and $\mathrm{S})$ range from -1 to -2 . The most characteristic chemical signature is, however, the extreme low carbon abundance in all these stars. The low metallicity, position in the HR-diagram and kinematics point to a late post-AGB evolution of low-initial-mass objects. The post-AGB nature is further strengthened by the detection of remnants of the circumstellar dust around a few objects and the very similar chemical patterns displayed around C-poor halo PN (Conlon et al. 1993). Similar objects were recently discovered towards the Galactic Centre (Venn et al. 1998). It is remarkable that the special chemical patterns displayed by these stars are not found among the F-type post-AGB stars ! 


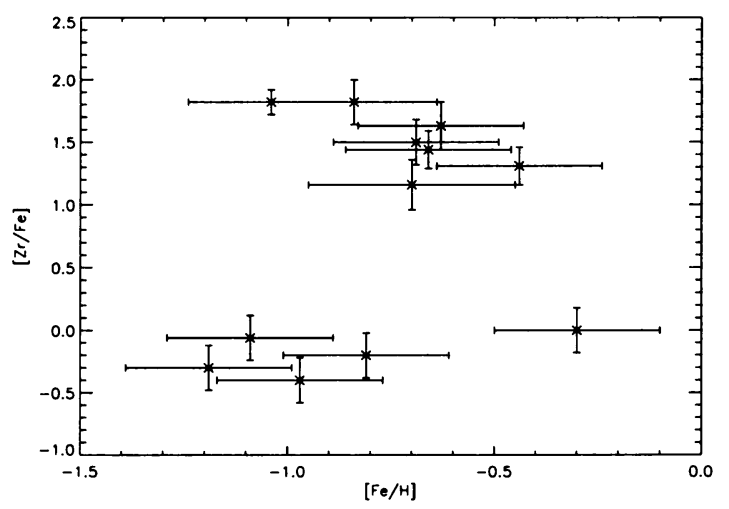

Figure 1. The $[\mathrm{Zr} / \mathrm{Fe}]$ abundance ratio as a measure of the 3rd dredgeup efficiency versus metallicity for post-AGB stars showing a double-peaked energy distribution. The enriched objects are the $21 \mu \mathrm{m}$ stars, while the lower ones are very similar objects but without chemical enrichment due to the 3 rd dredge-up.

\section{Binary post-AGB evolution}

In this section we focus on the optically bright post-AGB stars showing a broad IR excess consisting not only of a cool dust emission but also of a significant hot dust component which subsequently turned out to be binaries (see Sect. 4.3). If one assumes that the broad IR excess is caused by hot circumstellar dust in radiative equilibrium with the radiation field of the central object, the dust must be very close to the central star. Since in most objects there is no evidence for a significant present day post-AGB mass-loss, the dust must be stored somewhere in the system presumably in a circumbinary dusty disc (Waters et al. 1993). Photometric monitoring campaigns showed that the fundamental period in the photometry for some objects is the binary period and the photometric behaviour can be understood as caused by variable circumstellar reddening in the case of HR 4049 and HD 213985 (Waelkens et al. 1991; Waelkens et al. 1995) or in terms of a changing scattering angle during orbital motion for HD 44179 (Waelkens et al. 1996). The extrema of the photometry occur at conjunction.

While the formation of this circumstellar dust geometry is not clear, binary interactions during phases of heavy mass-loss must be involved.

\subsection{Case study: the Red Rectangle}

The best studied circumbinary disc is certainly the one in the centre of the famous X-shaped reflection nebula, the Red-Rectangle (RR, Cohen et al. 1975). It has recently become clear that many of the remarkable phenomena observed in the RR are closely related to the special binary nature of the central object HD 44179 and the presence of its circumbinary disc (e.g. Waelkens et al. 1996). The disc is resolved in ground-based high-spatial-resolution imaging at optical and near-IR wavelengths (e.g. Roddier et al. 1995; Osterbart et al. 1997) as well as in HST snapshots (Bond et al. 1997). The central object is not seen directly at optical wavelengths but only in scattered light escaping from the poles of the thick disc. We give a short overview of the observational characteristics linked to this binarity: the atmosphere of HD 44179 is severely depleted in re- 
fractory elements (Waelkens et al. 1992) making the star one of the most iron deficient objects known $([\mathrm{Fe} / \mathrm{H}]=-3.3$ ) in our Galaxy (see section 4.2 ); the extremely weak microwave $\mathrm{CO}$ emission with a small velocity width observed in the RR (Jura et al. 1995) together with the presence of very large grains inferred from $\mathrm{cm}$ continuum measurements (Jura et al. 1997) are distinct observational characteristics that can best be explained by long-term processing of dust in a stable circumstellar disc; the $\mathrm{CO}$ kinematics points to a Keplerian motion of this disc instead of a gas-outflow from the system. The stability of the disc was recently dramatically confirmed by the detection of crystalline silicates in this notorious carbon-rich nebula (Waters et al. 1998). The most likely scenario for the presence of oxygen-rich material is that the silicates in the long-lived disc were formed before HD 44179 became carbon-rich and expelled the C-rich nebula (Waters et al. 1998). Also the high crystallisation fraction of the silicates is a distinct signature of the strong processing of dust in stable disc-like geometries (Waters \& Molster in this volume).

Although the interacting binary nature of the Red Rectangle is now well established, the carriers of the unique presumably molecular emission features and nebular emission, for which the nebula is also famous (Schmidt et al. 1980), are still largely unknown.

\subsection{Depletion versus deficiency}

It is now generally accepted that the extremely low photospheric iron abundances down to $[\mathrm{Fe} / \mathrm{H}]=-4.8$ of some post-AGB stars do not reflect initial conditions but are acquired during stellar evolution. During this process, dust formation selectively removes elements with a high dust-condensation temperature from the gas phase and subsequent gas-dust separation and gas accretion leave the photosphere depleted of these refractories. This depletion process mimics 3rd dredge-up nucleosynthetic photospheric abundance patterns by increasing the $\mathrm{C} / \mathrm{O}$ ratio (since the $\mathrm{CO}$ molecule will be abundant in the gas-phase) and $\mathrm{C} / \mathrm{Fe}$, $\mathrm{N} / \mathrm{Fe}$ and $\mathrm{O} / \mathrm{Fe}$ abundance ratios, but can be identified by a high $\mathrm{S}$ abundance relative to adjacent $\alpha$-process elements and a high $\mathrm{Zn}$ abundance relative to other Fe-peak elements (Venn \& Lambert 1990; Bond 1991; Van Winckel et al. 1992). Since the s-process elements have high dust-condensation temperatures, they will also be depleted making the dredge-up history of depleted evolved atmospheres difficult to trace on the basis of photospheric spectra. IR circumstellar dust chemistry and molecular envelope chemistry are much more reliable tracers for these objects.

'Depleted' photospheric abundances therefore refer to the lack of refractory elements obtained by a chemical process during stellar evolution while 'deficient' abundances reflect sub-solar initial conditions or evolutionary decreased abundances from nucleosynthetic origin.

The binarity of all the extremely depleted objects (Van Winckel et al. 1995) and the observed presence of dust discs around some of them (e.g. Waelkens et al. 1991; Roddier et al. 1995), and the similar patterns observed in $\lambda$ Bootis stars (Venn \& Lambert 1990) led Waters et al. (1992) to suggest that the gasdust separation needed to account for the depletion pattern is likely to take place in a (long-lived) disc. Binarity is in this scenario for post-AGB stars, a necessary condition for the depletion process to be effective. 


\subsection{Orbital elements of binary post-AGB stars}

In Table 1 an overview is given of these post-AGB stars for which the binary period could be determined. The very wide spread in binary periods, in between 115 and 2600 days is apparent and since the broad IR excess is observed in all source (except $\mathrm{BD}+39.4926$ where no IR excess is observed till now and HD 46703 where only cool dust is detected) it seems that binaries with a large spread in orbital periods are able to store at least part of the dust expelled during phases of heavy mass-loss in the system. The dust disc stability implies that the time elapsed since these objects have left the AGB cannot be derived from the inner radius of the dust shell !

For the shorter-period binaries, the actual orbit is too small to accommodate a normal AGB star and the actual orbital period as well as the often surprisingly high eccentricity set strong constraints on previous evolution. It is not clear how these systems avoided fatal spiral-in on the AGB. The carbon-rich nature of the circumstellar environment around several sources is the best observational evidence that there has indeed been internal chemical enrichment. These systems are by no means the only evolved binaries where the same problem occurs since also the shorter-period binaries from the Ba-star group have very similar periods and eccentricities. For more detailed comparisons between these post-AGB orbital elements and other classes of evolved binaries we refer to the review by Jorissen in this volume.

It is clear that these stars represent a peculiar stage in binary evolution rather than a genuine post-AGB evolutionary stage. This binarity has very fundamental consequences on the evolution of the system since it affects not only the SED evolution but also the chemical evolution, the mass loss and mass-loss history, the gas/dust ratio of the circumstellar environment and even the dust processing (see also Waters \& Molster in this volume). Non-standard phenomena connected to the specific binary nature must therefore be explored to gain a better understanding of these systems.

\subsection{RV Tauri stars and binarity}

RV Tauri stars are relatively rare F, G or $\mathrm{K}$ variable supergiants characterised by alternating deep and shallow minima in their light curves (see also Lloyd Evans and Pollard \& Lloyd Evans in this volume). With their high luminosity and often large IR-excesses due to thermal radiation of circumstellar dust, there is general agreement that RV Tauri stars are low-mass objects in an evolved, probably post-AGB, evolutionary stage (Jura 1986; Pollard \& Lloyd Evans in this volume).

In a series of papers, Giridhar et al. (1994, 1998), Gonzalez et al. (1997a, $1997 \mathrm{~b}$ ) and Van Winckel et al. (1998b) have shown that the chemical composition of many field RV Tauri stars are also determined by depletion rather than dredge-up processes. If indeed binarity is a necessary condition for the depletion process to occur (see Sect. 4.2), the binary nature of these depleted RV Tauri stars should be explored. Recently ACHer, one of the prototypical RV Tauri stars, was indeed found to be a member of a wide binary system (Van Winckel et al. 1998) but binary motion is also observed in the RV Tauri stars IW Car (Pollard et al. 1996), EP Lyr (Gonzalez et al. 1997a), U Mon (Pollard $\&$ Cottrel 1995). Moreover ST Pup, a W Vir star showing also chemical deple- 
Table 1. Binary post-AGB stars for which the period has been determined. All the objects (except BD+39 4926 and HD 46703) show the presence of hot dust which is interpreted as due to a dusty disc in the systems. Note the large spread in binary periods. The upper part consists of those objects where photospheric patterns are determined by depletion, the lower part where no chemical analysis was performed yet or no depletion patterns could be identified. Besides the orbital period, eccentricity and mass function, the chemical type of the circumstellar material is indicated. References are 1) Kodaira et al. 1970; 2) Pollard et al. 1996; 3) Waters et al. 1993; 4) Van Winckel et al. 1995; 5) Van Winckel et al. 1998a; 6) Hrivnak 1998; 7) Van Winckel et al. 1998b; 8) Van Winckel et al. 1999

\begin{tabular}{|l|rrrr|}
\hline Name & $\begin{array}{r}\text { Period } \\
\text { days }\end{array}$ & e & $\begin{array}{r}\text { F(M) } \\
\mathrm{M}_{\odot}\end{array}$ & CS-chemistry \\
\hline HR 4049 & 429 & 0.31 & 0.143 & C-rich \\
HD 44179 & 318 & 0.38 & 0.049 & C+O-rich \\
${\text { HD } 52961^{8}}^{4}$ & 1305 & 0.3 & 0.46 & C-rich \\
BD+39.4926 & 775 & & & $?$ \\
HD 213985 & 259 & 0.0 & 0.97 & C+O-rich \\
HD 46703 & 600 & 0.34 & 0.28 & $?$ \\
AC Her & 1194 & 0.12 & 0.25 & O-rich \\
\hline SAO 1733295 & 115.9 & 0.0 & 0.026 & $?$ \\
89 Her $^{3}$ & 288.4 & 0.19 & 0.00083 & O-rich \\
HD 95767 & \pm 2050 & \pm 0.3 & & $?$ \\
HD 131356 & 1534 & 0.3 & 0.72 & $?$ \\
U Mon $^{2}$ & 2597 & 0.43 & 0.92 & O-rich \\
\hline
\end{tabular}

tion patterns, is also a binary with a period of 410 days and the only B-type post-AGB star with a high $\mathrm{S}$ abundance relative to the other $\alpha$-elements, is also a binary (Hambly et al. 1996).

Since large-amplitude pulsational radial-velocity variations and strong lineasymmetries and even line-splitting at some pulsational phases render direct detection of orbital motion cumbersome for RV Tauri stars, alternative tracers of the possible binary nature of these stars should be explored. One of the possibilities is a detailed analysis of the SED. Lloyd Evans (this volume) has shown that the dust-envelope of RV Tauri stars are characterised by a considerable near-IR excess. This disc-like appearance is very similar to the stars discussed in Sect. 4.3 which are known to be binaries.

Another possibility is a detailed analysis of the dust and molecular emission of the circumstellar material. The best studied example is ACHer for which there is strong observational evidence from $\mathrm{CO}$ rotational line flux and velocity measurements, sub-mm continuum measurements and from a full 2.3-40 $\mu \mathrm{m}$ SWS ISO spectrum that it is also surrounded by a long-lived dust-disc (Van Winckel et al. $1998 \mathrm{~b}$ and references therein) very similar to the oxygen-rich dust-disc observed in the Red Rectangle (Waters \& Molster in this volume). In 


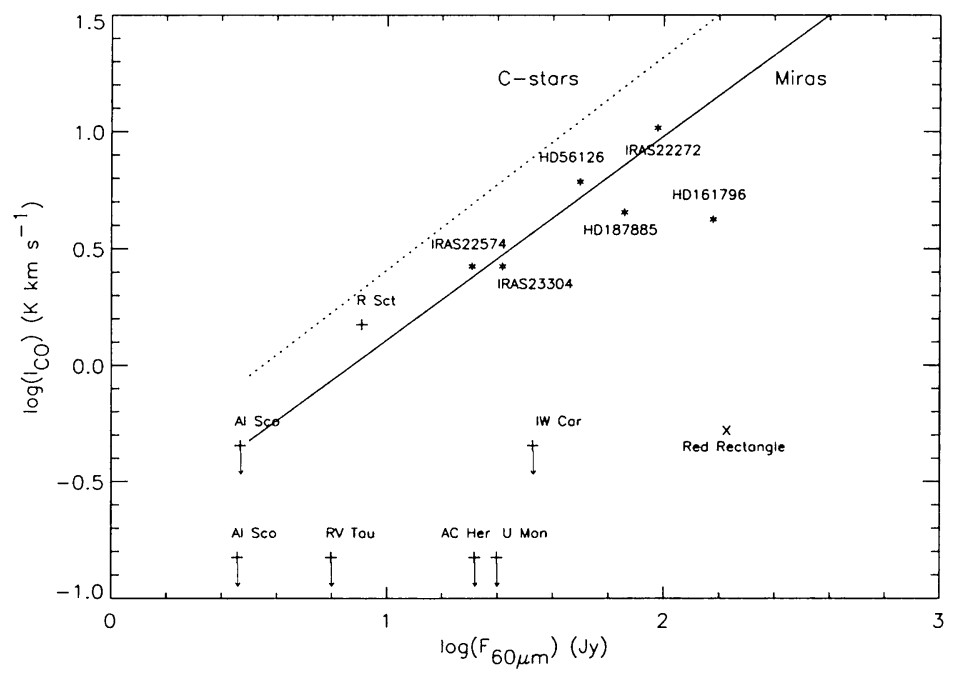

Figure 2. The $\mathrm{CO}(1-0)$ microwave integrated intensity versus the $60 \mu \mathrm{m}$ flux both in log of post-AGB objects. The relation for C-stars (O-rich Miras) as determined by Nyman et al. (1992) is given by a dotted (full) line. The asterisks give the positions of double-peaked post-AGB stars, the +-signs indicate the RV Tauri stars. The arrows indicate upper limits. Data are from Bujarrabal et al. 1988; Alcolea et al. 1991; Loup et al. 1993; van der Veen et al. 1993; Jura et al. 1995

order to investigate the molecular emission of the circumstellar environment of RV Tauri stars we compiled in Fig. 2 microwave $\mathrm{CO}$ emission measurements of these stars and of the double-peaked post-AGB stars. It clearly shows that the circumstellar environment around RV Tauri stars is deficient in CO while other known and suspected single post-AGB stars like the $21 \mu$ m-objects or HD 161796 do follow the $\mathrm{CO} / 60 \mu \mathrm{m}$ relation known for AGB objects confirming that in the latter objects the IR excess is formed by the ejected AGB superwind. A similar and even stronger CO deficiency is observed in the Red Rectangle (Jura et al. 1995). The depletion is clearly reflected in the gas/dust ratio of the cool circumstellar dust envelope. This indicates again that the depletion process is intimately related to the special geometry of the circumstellar material.

Assuming that indeed the circumstellar material is trapped in the system, it is quite possible that the viewing angle onto the binary orbit determines the photometric class of the RV Tauri star: objects with a low orbital inclination would be the RVa objects with their constant mean magnitude, while those with a high inclination so that the disc is seen nearly edge-on would be the class $\mathrm{RVb}$ objects where the variability of the mean magnitude is due to variable circumstellar extinction during orbital motion.

The commonly observed photospheric chemical depletion pattern, the peculiar SEDs and the generally weak and narrow microwave $\mathrm{CO}$ emission of RV Tauri stars together with the difference in photospheric class of the RV Tauri objects can all be naturally explained by assuming that the circumstellar material is not freely expanding, but trapped in the binary system. 


\section{Conclusions}

We can conclude that the $21 \mu \mathrm{m}$ objects are the successors of the carbon stars. The wide spread in integrated neutron exposure they display together with the absence of 3rd dredge-up yields in the photospheres of very similar stars is still not well understood.

There is strong observational evidence that the final evolution of binary stars with a very wide period distribution (from 116 to 2600 days) is strongly affected by this binarity. The fundamental property seems to be that at least part of the circumstellar material is trapped in a circumbinary disc which has a strong impact on the chemical and dynamical evolution of the system. There is still good observational evidence that depletion of refractory elements only occurs in binaries. The high pole to orbital-plane density gradient due to the dusty disc in these binaries will certainly affect also future evolution during the PN phase. Waters \& Molster (in this volume) shows that also the dust evolution and processing is very different from the processing in normal outflows.

Since binaries display a prolonged IR lifetime due to the presence of this stable circumbinary dusty disc, any sample of post-AGB objects defined on IR measurements is bound to be biased towards binaries. Also in the large sample of post-AGB stars with a very weak optical counterpart, binaries must be present. The best candidates are those with a very large pole-to-equator density gradient in the circumstellar envelope.

Acknowledgments. The many instructive discussions with Rens Waters and Christoffel Waelkens are warmly acknowledged. HVW is post-doctoral fellow of the Fund for Scientific Research, Flanders (Belgium)

\section{References}

Alcolea J., Bujarrabal V., 1991, A\&A 245, 499

Bakker E.J., Waters L.B.F.M., Lamers H.J.G.L.M., et al., 1996, A\&A 310, 893

Bakker E.J., Van Dishoeck E.F., Waters L.B.F.M., Schoenmaker T., 1997, A\&A 323, 469

Bakker E.J., Lambert D.L., 1998, ApJ 502, 417

Blöcker T., 1995, A\&A 299, 755

Bond H.E., 1991, IAU Symp. 145, p. 341

Bond H.E., Fullton L.K., Schaefer K. et al., 1997, IAU Symp. 180, p. 211

Bujarrabal V., Bachiller R., Alcolea J., Martín-Pintado, 1988, A\&A 206, L17

Cohen M., Anderson C.M., Cowley A., et al., 1975, ApJ 196, 179

Conlon E.S., Dufton P.L., Keenan F.P., McCausland R.J.H., 1991, MNRAS 248, 820

Conlon E.S., Dufton P.L., Keenan F.P., et al., 1993, A\&A 272, 243

Dayal A., Hoffmann W.F., Bieging J.H., et al., 1998, ApJ 492, 603

Decin L., Van Winckel H., Waelkens C., Bakker E.J., 1998, A\&A 332, 928

Giridhar S., Rao N.K., Lambert D.L., 1994, ApJ 437, 476

Giridhar S., Lambert D.L., Gonzalez G., 1998, ApJ in press

Gonzalez G., Wallerstein, G., 1996, MNRAS 280, 515

Gonzalez G., Lambert D.L., Giridhar S., 1997a, ApJ 479, 427

Gonzalez G., Lambert D.L., Giridhar S., 1997b, ApJ 481, 481

Hambly N.C., Dufton P.L., Keenan F.P., Lumsden S.L., 1996, MNRAS 278, 811

Hrivnak B.J., 1995, ApJ 438, 341 
Hrivnak B.J., 1998, in "Carbon Stars", IAU Symp. 177, in press

Hrivnak B.J., Kwok S., Volk K.M., 1989, ApJ 346, 265

Jura M., 1986, ApJ 309, 732

Jura M., Balm S.P., Kahane C., 1995, ApJ 453, 721

Jura M., Turner J., Balm S.P., 1997, ApJ 474, 741

Klochkova V.G., 1995, MNRAS 272, 710

Kodaira K., Greenstein J.L., Oke J.B., 1970, ApJ 159, 485

Kwok S., Volk K.M., Hrivnak B.J., 1989, ApJ 345, 51

Loup C., Forveille T., Omont A., Paul J.F., 1993, A\&AS 99, 291

Luck R.E., Bond H.E., Lambert D.L., 1990, ApJ 357, 188

McCausland R.J.H., Conlon E.S., Dufton P.L., Keenan F.P., 1992, ApJ 394, 298

Meixner M., 1997, IAU Symp. 180, p. 325

Meixner M., Skinner C.J., Graham J.R., et al., 1997, ApJ 482, 897

Moehler S., Heber U., 1998, A\&A 335, 985

Napiwotzki R., Heber U., Köppen J., 1994, A\&A 292, 239

Nyman L.- $\AA$., Booth R.S., Carlström U.U., et al., 1992, A\&AS 93, 121

Omont B.E., Moseley S.H., Cox P., et al., 1995, ApJ 454, 819

Osterbart R., Langer N., Weigelt G., 1997, A\&A 325, 609

Oudmaijer R.D., van der Veen W.E.C.J., Waters L.B.F.M., et al., 1992, A\&AS 96, 625

Pollard K.R., Cottrel P.L., 1995, ASP Conf. Ser. 83, 409

Pollard K.R., Cottrell P.L., Kilmartin P.M., Gilmore A.C., 1996, MNRAS 279, 949

Pottasch S.R., Parthasarathy M., 1988, A\&A 192, 182

Reddy B.E., Parthasarathy M., Gonzalez G., Bakker E.J., 1997, A\&A 328, 331

Roddier F., Roddier C., Graves J.E., Northcott M.J., 1995, ApJ 443, 249

Schmidt G.D., Cohen H., Margon B., 1980, ApJ 239, L133

Skinner C.J., Meixner M.M., Hawkins, G.W., et al., 1994, ApJ 423, L135

Szczerba R., Omont A., Volk K., Cox P., Kwok S., 1997, A\&A 317, 859

Trams N.R., Waters L.B.F.M., Waelkens C., et al., 1991, A\&AS 87, 361

van der Veen W.E.C.J., Trams N.R., Waters L.B.F.M., 1993, A\&A 269, 231

Van Winckel H., 1997, A\&A 319, 561

Van Winckel H., Mathis J.S., Waelkens C., 1992, Nature 356, 500

Van Winckel H., Waelkens C., Waters L.B.F.M., 1995, A\&A 293, L25

Van Winckel H., Waelkens C., Waters L.B.F.M., 1996, A\&A 306, L37

Van Winckel H., Waelkens C., Waters L.B.F.M., 1998a, IAU Symp. 177, in press

Van Winckel H., Waelkens C., Waters L.B.F.M., et al., 1998b, A\&A 336, L17

Van Winckel H., Waelkens C., Fernie J.D., Waters L.B.F.M., 1999, A\&A, accepted

Venn K.A., Lambert D.L., 1990, ApJ 363, 234

Venn K.A., Smartt S.J., Lennon D.J., Dufton P.L., 1998, A\&A 334, 987

Waelkens C., Lamers H.J.G.L.M., Waters L.B.F.M., et al., 1991, A\&A 242, 433

Waelkens C., Van Winckel H., Trams N.R., Waters L.B.F.M., 1992, A\&A 256, L15

Waelkens C., Waters L.B.F.M., Van Winckel H., Daems K., 1995, Ap\&SS 224, 357

Waelkens C., Van Winckel H., Waters L.B.F.M., Bakker E.J., 1996, A\&A 314, L17

Waters L.B.F.M., Trams N.R., Waelkens C., 1992, A\&A 262, L37

Waters L.B.F.M., Waelkens C., Mayor M., Trams N.R., 1993, A\&A 269, 242

Waters L.B.F.M., Waelkens C., Van Winckel H., 1997, IAU Symp. 180, p. 211

Waters L.B.F.M., Waelkens C., Van Winckel H., et al., 1998, Nature 391, 868

Začs L., Klochkova V.G., Panchuk V.E., 1995, MNRAS 275, 764

Začs L., Klochkova V.G., Panchuk V.E., Spēlmanis R., 1996, MNRAS 282, 1171 